\title{
NONLINEAR DYNAMICS OF BROAD-BAND LASERS
}

\author{
FINAL REPORT
}

for Period 9/15/90--9/14/91

\author{
Michael G. Raymer \\ Department of Physics \\ University of Oregon \\ Eugene, OR 97403 \\ March 1991 \\ U.S. DEPARTMENT OF ENERGY \\ AGREEMENT NO. DE-FG06-88ER13973
}

\section{NOTICE}

This report was prepared as an arcount of work sponsored by the U.S. Government. Neither the United States nor the Department of Energy, nor any of their employees, nor any of their contractors, subcontractors, or their employess, make any warranty, express or implied, or assumes any legal liability or responsibility for the accuracy, completeness, or usefulness of any information, apparatus, product or process disclosed or represents that its use would not infringe privately-owned rights. 


\section{List of Publications}

1. "Instabilities and chaos in a multimode, standing-wave $\mathrm{cw}$ dye laser," I.A.McMackin, M.Beck, C.Radzewicz, and M.G.Raymer, Phys. Rev. A 38, 820 (1988).

2. "Strong-field dynamics of a multimode, standing-wave dye laser," M.G.Raymer, Z.Deng, and M.Beck, J.Opt.Soc.Am. B5, 1588 (1988).

3. "Transition from quantum-noise-driven dynamics to deterministic dynamics in a multimode laser," M.Beck, I.McMackin, and M.G.Raymer, Phys. Rev. A 40, 2410 (1989).

4. "Conical Emission from a micro-cavity dye laser," S. Hodges, W. Gadornski, and M.G. Raymer, Proceedings of the 1990 International Quantum Electronics Conference, May 1990.

5. "Dynamics of Multimode, Short-Cavity Dye Lasers," W.Gadomski, S.E.Hodges, and M.G.Raymer, Proceedings of Nonlinear Dynamics in Optical Systems, June,1990.

6. "Deterministic and quantum noise in dye lasers," M.G.Raymer, W.Gadomski, and S.E.Hodges, Proceedings of SPIE Symposium on Laser Noise, Nov. 5-6, 1990. 


\section{ABSTRACT}

A number of advances were made in improving the understanding of multimode dye laser dynamics. The source of mode-intensity fluctuations in a three-mirror, multimode dye laser has been identified in the typical operating regime well above threshold. It was found experimentally that deterministic four-wave mixing interactions are the underlying cause. We have also made new theoretical studies concerning the regime just above threshold and predicted that the mode fluctuations will be dominated by quantum noise in this regime. We have constructed a short-cavity dye laser in order to enhance this quantum effect, and have made measurements of the turn-on time statistics of individual modes. Effects of coherent, fourwave mixing interactions on these statistics have been identified. 


\section{NONLINEAR DYNAMICS OF BROAD-BAND LASERS}

Following the invention of the laser, much of the theoretical foundation of quantum-noise effects was laid down in the 1960's, by Haken [1], Lamb [2], and Lax [3]. The understanding of deterministic, chaotic behavior in lasers, however, did not begin to mature until the 1980 's when the wide availability of fast computers made possible the numerical simulation of a range of complex phenomena. Also at that time the advanced technology of lasers and computer-based data-collection systems made possible the detailed experimental characterization of nonlinear optical systems.

The present research project has focussed on understanding multimode lasers under the simultaneous influence of quantum noise and deterministic, chaotic dynamics. At a recent SPIE Symposium [4] on this topic the $\mathrm{Pl}$ reviewed our studies of $\mathrm{cW}$ multimode dye lasers.

\section{A. Classification of lasers by dynamics}

Lasers can be classified according to their dynamical behavior. Define:

$$
\begin{aligned}
& \gamma_{c}=\text { Laser cavity decay rate } \\
& \gamma_{1}=\text { Population inversion decay rate } \\
& \gamma_{2}=\text { Medium electronic polarization decay rate }
\end{aligned}
$$

Then the classes follow from the relations between these rates:

CLASS A - $\gamma_{2} \gg \gamma_{1} \gg \gamma_{c}:$ No relaxation oscillations (long-cavity dye laser; color center)

CLASS B -- $\gamma_{2} \gg \gamma_{1} \sim \gamma_{c}$ : Relaxation oscillations (short-cavity dye laser; Ti:S; semiconductor laser; Nd-YAG)

CLASS C -- $i_{2} \sim \gamma_{1}-\gamma_{c}:$ Lorenz-Haken chaos [5] (far IR $\mathrm{NH}_{3}$ laser)

B. Noise and dynamics in multimode dye lasers

Typical $\mathrm{cw}$ dye lasers are Class $\mathrm{A}$ since the inversion lifetime is several nsec and the cavity lifetime must be large ( 50-100 nsec) for the laser to be above threshold. In addition the polarization decay time is less 
than 100 fsec. Thus they cannot display relaxation oscillations of the inversion and the optical field. They have, however, been found to have several interesting behaviors.

Two-mode ring dye lasers are known to undergo spontaneous switching between quasi-stable, opposite-traveling modes.[6] The switching, which occurs randomly, is ultimately caused by quantum (spontaneous emission) noise, although pump fluctuations also play an important role. This illustrates that microscopic quantum noise can have a dramatic impact on the operating states of a laser at the macroscopic level.

The two-mode ring dye laser is well described by an equation of motion for the slowly varying electric-field amplitudes $E_{n}$ for each mode, ie

$$
\frac{d}{d t} E_{n}=\left[-\gamma_{n}+g_{n}-\sum_{j=1}^{N} \xi_{n j}\left|E_{j}\right|^{2}\right] E_{n}+F_{n}(t)
$$

where $\mathrm{N}=2$ (two modes), $\gamma_{n}$ is the cavity-decay rate for the $n$-th mode, $g_{n}$ is the unsaturated gain coefficient for the $n$-th mode, the sum includes self gain saturatio' 1 and cross saturation (gain competition) by the modes, and $F_{n}(t)$ is a delta-correlated random noise (Langevin term) representing spontaneous emission into the n-th mode. Any noise present in the pumping rate will appear as a modulation of the gain coefficient. The switching between modes can be pictured as a random walk between two stable minima in a potential function, separated by a barrier.

Multimode cw dye lasers were studied by our group to determine if quantum-induced switching plays a role in these more complex systems. $[4,7]$ With a typical three-mirror, folded cavity with length $50 \mathrm{~cm}$ we found, in agreement with previous studies, $[8,9,10]$ that the modec nave no quasistable behavior--rather they continuously exchange energy in a seemingly random fashion. An example of our data, showing the time dependence of the intensity of a single mode out of about 30 is shown in Fig.1. That these fluctuations are truly a multimode effect is indicated by the fact that the total output intensity of the laser is nearly constant. Our group sinde the first quantitative measurements of the correlation time of the individunlmode intensity fluctuations, and the results are 

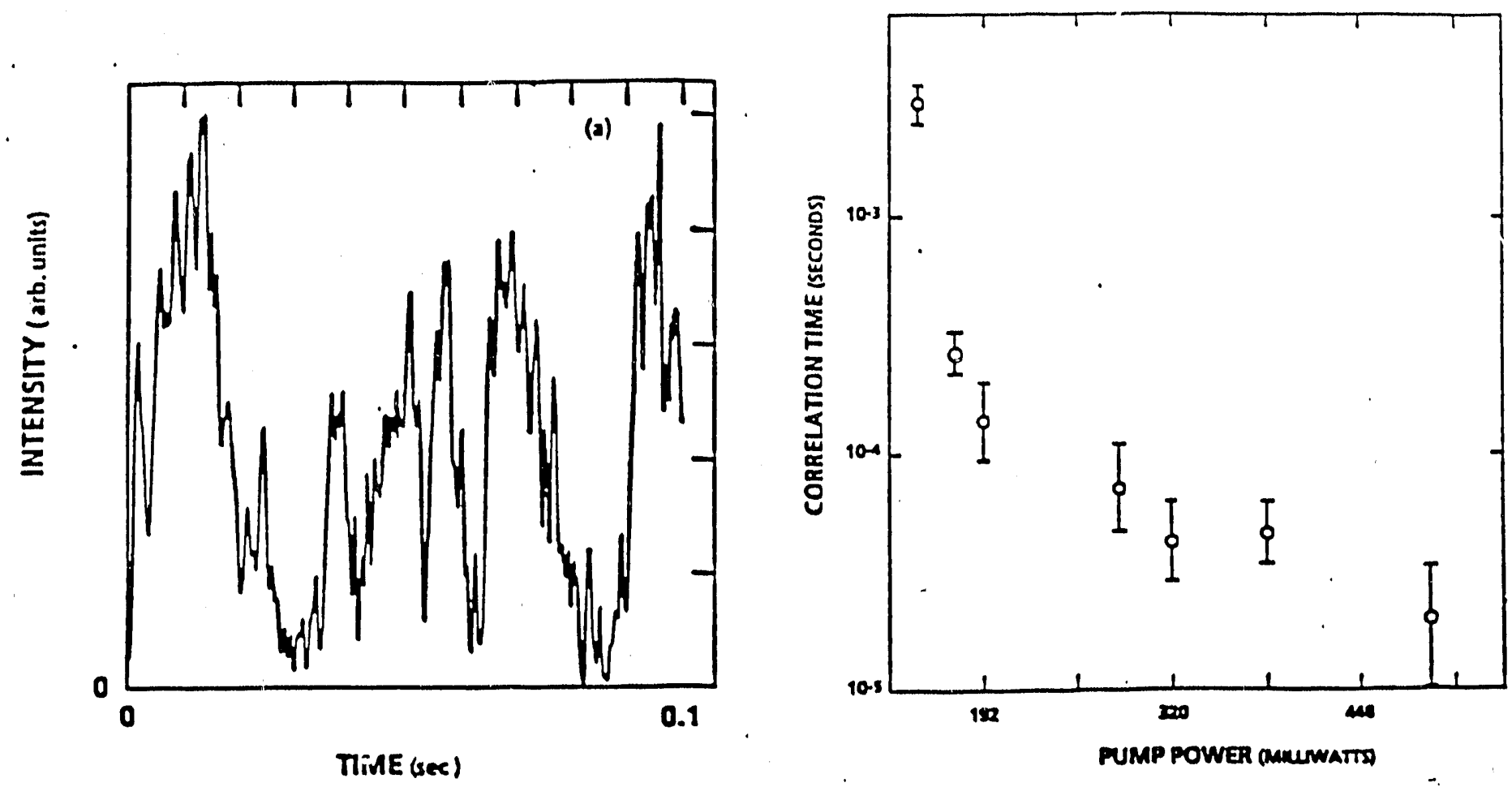

Fig.1--Intensity of individual mode of multimode dye laser.(left) Intensity correlation time of mode versus pump power.(right)

shown versus pump power in Fig.1. Note that the correlation time is very long compared with any obvious time scale for the medium or cavity.

The observed trend of decreasing correlation time with increasing pump is in contradiction with the prediction made by simply extending Eq.(1) to the case of many modes, as done in [11,12]. The reason, we pointed out,[7] is the absence in Eq.(1) of coherent mode coupling due to fourwave mixing involving spatial gratings in the gain medium. To include this in the model we generalized Eq.(1) to read

$$
\frac{d}{d t} E_{n}=\left[-\gamma_{n}+g_{n}-\sum_{j=1}^{N} \xi_{n j}\left|E_{j}\right|^{2}\right] E_{n}+g_{n} \sum_{l \neq n} \sum_{m} D_{n m l} E_{l} E_{m} E_{m+l-n}^{*}+F_{n}(t)
$$

where the double sum accounts for coherent interactions between groups of four modes. The coupling coefficients $D_{n m l}$ are determined by the frequencies and spatial distributions of the modes involved. Modes closer 
in frequency interact more strongly because the molecules can better respond to the intermode beating that creates the coherent spatial gratings responsible for the interaction.

By solving Eq.(2) numerically without quantum noise $\left(F_{n}(t)=0\right)$ we found that full-scale fluctuation of mode amplitudes arises from deterministic effects, independently of stochastic effects. A typical solution is shown in Fig.2. Furthermore, we found that the trend of
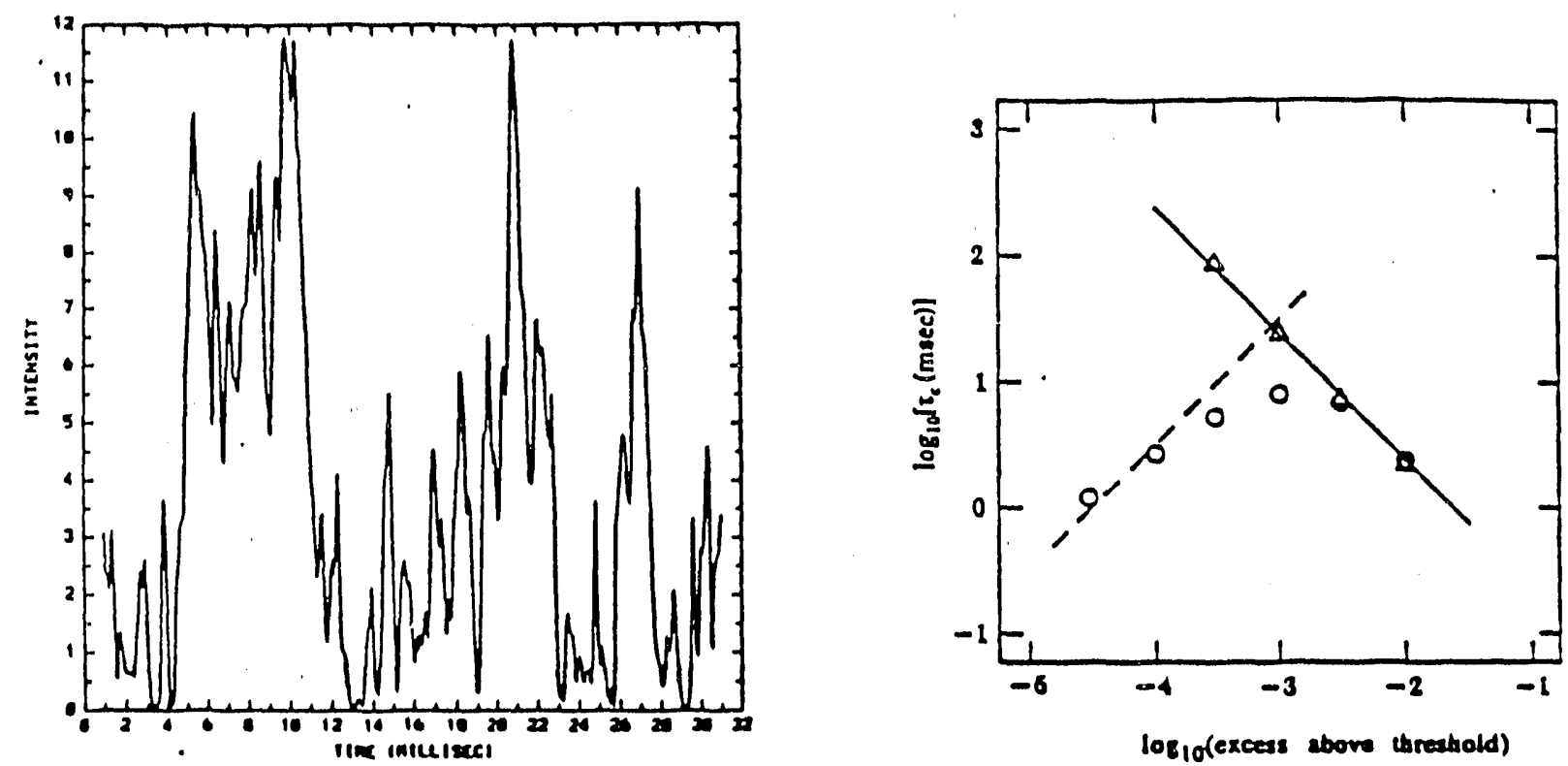

Fig. 2-- Solution of Eq. 2 for individual mode without noise added.(left) Intensity correlation time from Eq.2, with (circles) and without (triangles) quantum noise added. (right)

decreasing correlation time is properly described by this theory. Evidently the four-wave mixing leads to a continual exchange of energy between the modes, the time scale of which decreases when the intensities are higher as expected for a nonlinear optical process.

As a further confirmation that the dynamics are deterministic, we employed the data-analysis algorithm of Grassberger and Procaccia [13] to find the "correlation dimension" of the strange attractor underlying the dynamics. We found, as did the group of Atmanspacher,[14] a low dimension, indicating that the typical $\mathrm{cw}$, multimode dye laser displays deterministic evolution, possibly chaos. 
However, this leaves a gap in our understanding of Class A multimode lasers--that is, where are the effects of quantum noise in these systems? To answer this question we solved Eq.(2) numerically with quantum noise,[15] and the results are shown in Fig.2. The triangles (and solid line) show again the result without noise and the circles (and dashed line) show the solution with noise. It is seen that well above threshold, where the experiments were done, the correlation time is predicted to decrease with increasing pump power, in agreement with experiment. On the other hand, very close to threshold quantum noise decreases the correlation time as threshold is approached. This is in agreement with the old quantum theories, $[11,12]$ which neglected coherent mode coupling. The region very near threshold is difficult to study experimentally due to pump fluctuations.

Short-cavity dye lasers are the subject of our recent research because they offer the possibility to study a laser in which both quantum noise and coherent mode coupling play a significant role. This arises because in a short cavity the free-spectral range separating the modes is large, weakening the coherent coupling relative to the noise which is independent of cavity length. Short-cavity dye lasers are Class B since the cavity round-trip time is small. Thus the theory in Eq.(2) is not valid for describing their dynamics.

Mode turn-on statistics have been measured by our group for a short-cavity dye laser (length $2.5 \mathrm{~cm}$ ) following sudden turn on of the pump power.[16] This provides a way to observe the effects of quantum noise in the presence of pump fluctuations since the turn-on dynamics occur on a time scale short compared to the pump variations.[17] To attempt to unravel the effects of coherent mode coupling from quantum noise, we studied both a laser with "small" mode spacing $\left(30 \mathrm{~cm}^{-1}\right)$ and a laser with larger spacing $\left(300 \mathrm{~cm}^{-1}\right)$ determined by intracavity etalons.

For the large mode spacing we obtained the data shown as points in Fig.3 which shows several realizations of the random turn-on behavior. The solid curves are the result of stochastic simulations of a rate-equation theory that includes quantum noise but no coherent mode coupling. The agreement appears satisfactory. On the other hand, for the small mode spacing signiticant differences in 

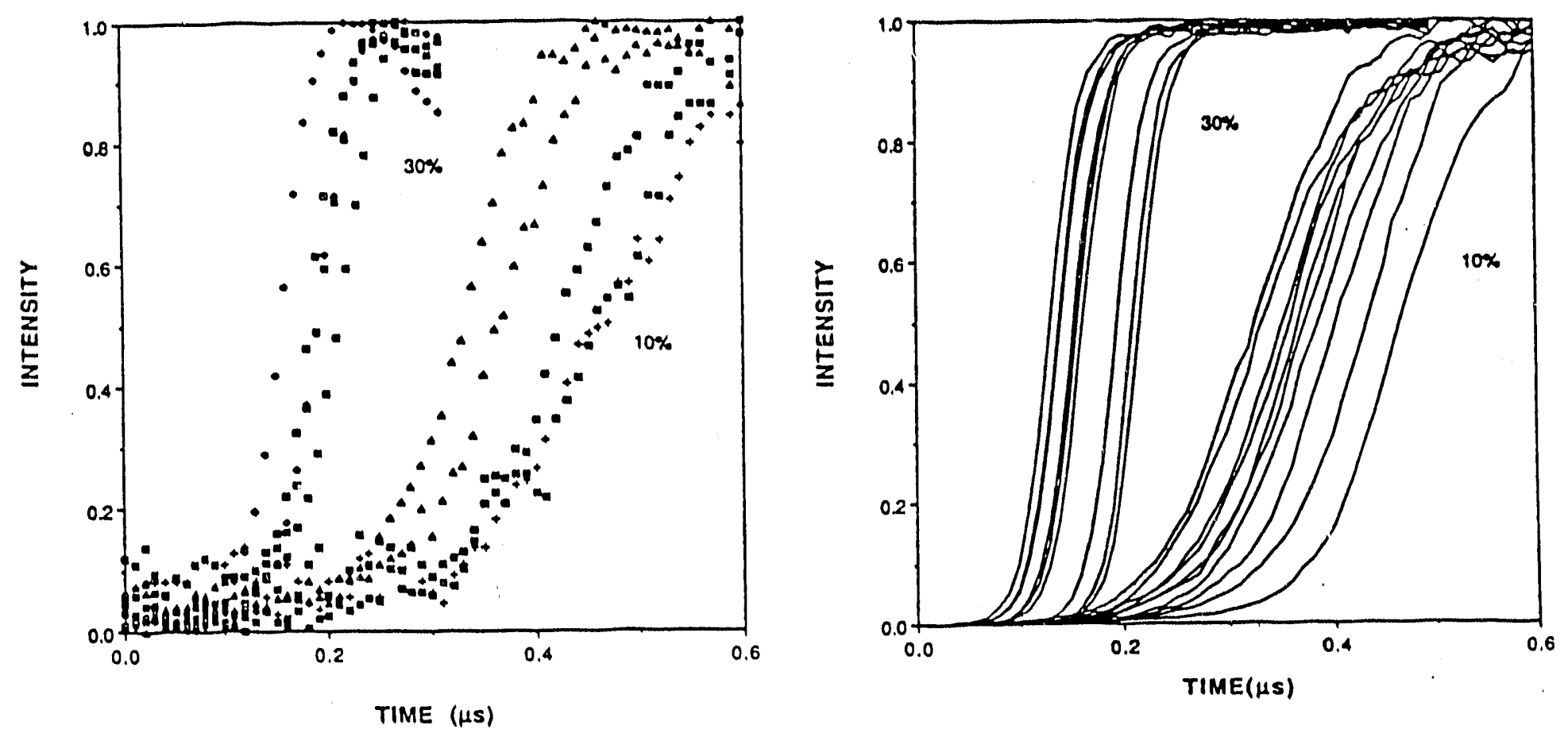

Fig.3-- Ten realizations of individual-mode turn on, for $10 \%$ and $30 \%$ above threshold; experiment (left) and theory (right) for 3-5 mode laser.

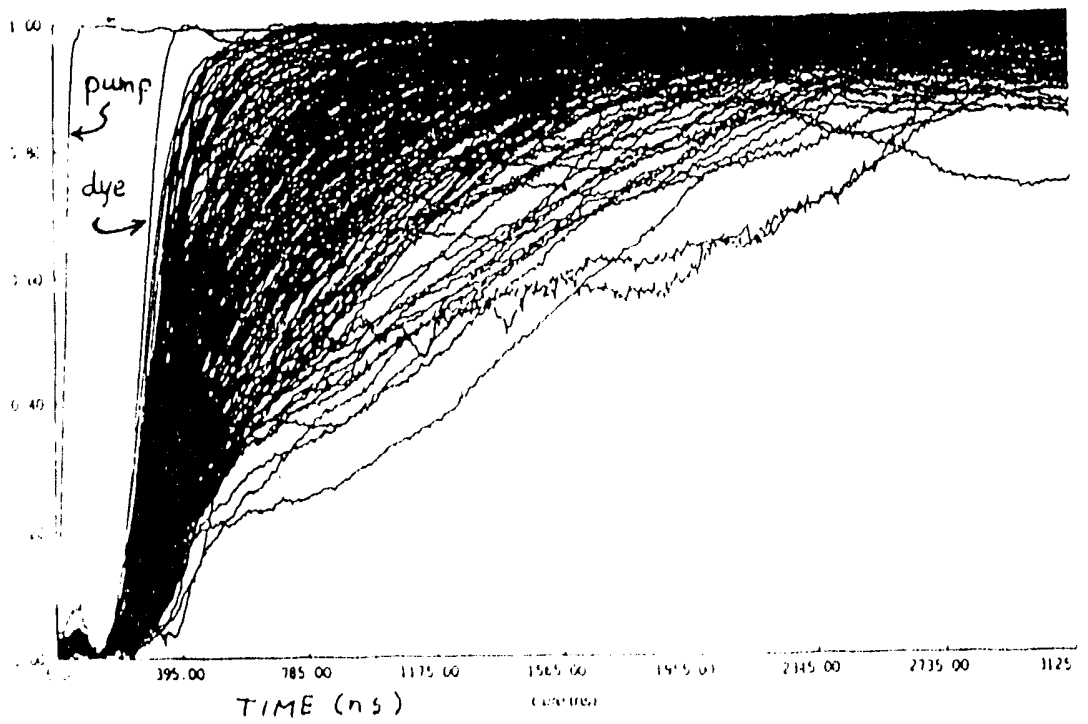

Fig.4-- Experimental realizations of mode turn on in a 30-mode laser. 
qualitative behavior of the modes were observed (overshoot, very slow turn on), as shown in Fig.4, which shows 256 realizations of turn-on behavior. As will be discussed below in the theory section, we believe that these effects are caused by coherent mode coupling.

\section{Theory of Class B lasers}

An important element in the success of this program is the development of a predictive theory of multimode, Class B lasers, in which the cavity lifetime is comparable to or less than the population lifetime. As mentioned above, in this case the population cannot be adiabatically eliminated from the equations as was done in Eq.(2). We have succeeded in deriving in this case a new set of equations of motion for the mode amplitudes $E_{n}$ and the different spatial components of the population inversion, $W_{n j}(t)$, defined by

$$
W(z, t)=\sum_{n, j} W_{n j}(t) \cos (j \pi z / L) e^{i n(\pi / L) t}
$$

where $W(z, t)$ is the population inversion and $L$ is the cavity length. The coupled equations of motion are found to be

$$
\begin{aligned}
& \frac{d}{d t} W_{o j}=-\gamma_{1} W_{o j}-\sum_{k l} B_{j k l} W_{o k}\left|E_{l}\right|^{2}+\sum_{q} W_{o q} \sum_{m k l} D_{\text {qjmkl }} E_{l} E_{m-l+k} E_{k}^{*} \\
& \frac{d}{d t} E_{n}=(1-i \alpha)\left[-\gamma_{n}+\sum_{j} C_{n j} W_{o j}\right] E_{n}+\sum_{j} W_{o j} \sum_{m k} D_{n j m l} E_{l} E_{n-l+k} E_{k}^{*}+F_{n}(t) .
\end{aligned}
$$

where $\gamma_{1}$ is the population decay rate and $\alpha$ is the linewidth enhancement factor arising from amplitude-phase coupling.[18] These equations are similar to Eq.(2) except that the population inversion is now a dynamical variable. This allows for relaxation oscillations, in addition to coherent mode coupling.

Numerical solutions of Eq.(4) show behavior qualitatively similar to that found in our experiments. Figure 5 shows typical solutions for parameters similar to those in the short-cavity dye laser whose dynamics 
are shown in Fig.4. The slow turn-on behavior seen for some of the shots is seen in both the theory and the experiment in the case of the smaller mode spacing. We conclude that this behavior is due to the coherent fourwave mixing among modes, which is enhanced in this case.

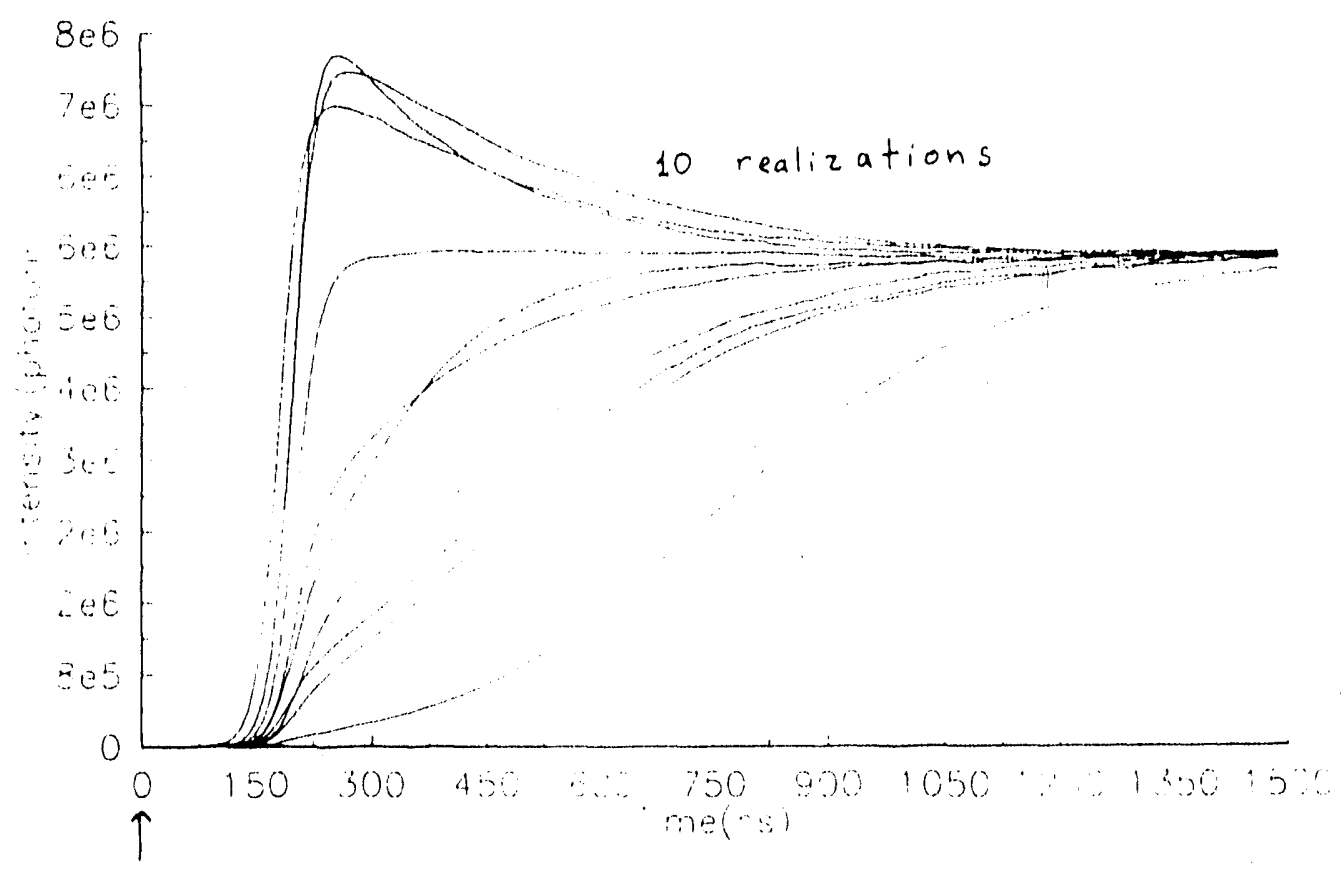

Fig.5-- Theoretical realizations of mode turn on in a 30-mode laser.

\section{Conical emission from short-cavity lasers}

We have designed and constructed a dye laser with cavity length that is adjustable from $50 \mu \mathrm{m}$ to a few $\mathrm{mm}$. In this laser an unexpected spatial distribution of the output of the short-cavity laser has been observed. Intense, sharp rings were found surrounding the usual lasing spot. In the case of a cavity with plane-parallel mirrors, the rings were founa to be at angles expected for a Fabry-Perot interferometer if the frequencies are those of the longitudinal cavity modes. That these frequencies are those occuring in the rings was verified by use of a monochromator. It appears that light in the lasing longitudinal modes is being scattered by something (mirrors, dirt, dye) inside the cavity and are constructively interfering only at the observed angles. It is not clear if the rings are lasing or just scattering passively. A conical laser emission similar to this was predicted in a famous paper by Kastler in 1962, but no observations have been reported. [19] 
It is not clear whether the conical emission affects in a significant way the mode-intensity dynamics in short-cavity lasers, which are of interest here.

\section{References}

1 H. Haken, Laser Theory (Springer Verlag, Berlin, 1984).

2 Laser Physics, M.Sargent, M.Scully, and W.Lamb (Addison Wesley, London, 1974).

3 See Quantum Statistical Properties of Radiation ,W. Louisell (Wiley, 1973).

4 M. Raymer, invited talk in Session on Laser Noise, at the SPIE Symposium on Laser Science and Optics Applications, Boston, Nov. 4-9 (1990).

5 H. Haken, Phys.Lett, 53A, 77 (1975).

6 P. Lett and L. Mandel, JOSA B 21615 (1985).

7 I.A.McMackin, M.Beck, C.Radzewicz, and M.G.Raymer, Phys. Rev. A 38, 820 (1988)

8 L.A.Pakhomycheva, E.A.Sviridenkov, A.F.Suchkov, L.V.Titova, and S.S.Churilov, JETP Lett. 12,43 (1970).

9 T.W.Hansch, A.L.Schawlow, and P.E.Toschek, IEEE J.Q.E. 6, 802 (1972).

10 V.M.Baev, T.P.Belikova, E.A.Sviridenkov, and A.F.Suchkov, Sov. Phys. JETP 47,21 (1978).

11 F.T.Hioe, J. Math. Phys. 19, 1307 (1978).

12 S.A.Kovalenko, Sov. J. Quant. Electron. 11, 759 (1981).

13 D.Grassberger and I.Procaccia, Phys Rev. A 28, 2591 (1983).

14 H. Atmanspacher, H. Scheingraber, and C. Vidal, Phys. Rev. A 32, 254 (1985); H. Atmanspacher and H. Scheingraber, Phys. Rev. A 34, 253 (1986).

15 M.Beck, I.McMackin, and M.G.Raymer, Phys. Rev. A 40, 2410 (1989).

16 M. G. Raymer, W. Gadomski, S. Hodges. and D. Adkison, in ref.4

17 S. Zhu, A. W. Yu, and R. Roy, Phys. Rev. A 34, 4333 (1986); F. de

Pasquale, J. M. Sancho, M. San Miguel, and P. Tartaglia, Phys. Rev. Lett.

562473 (1986).

18 C. Henry, IEEE JQE 18 259(1982).

19. A. Kastler, Appl. Opt.1,17(1962). 

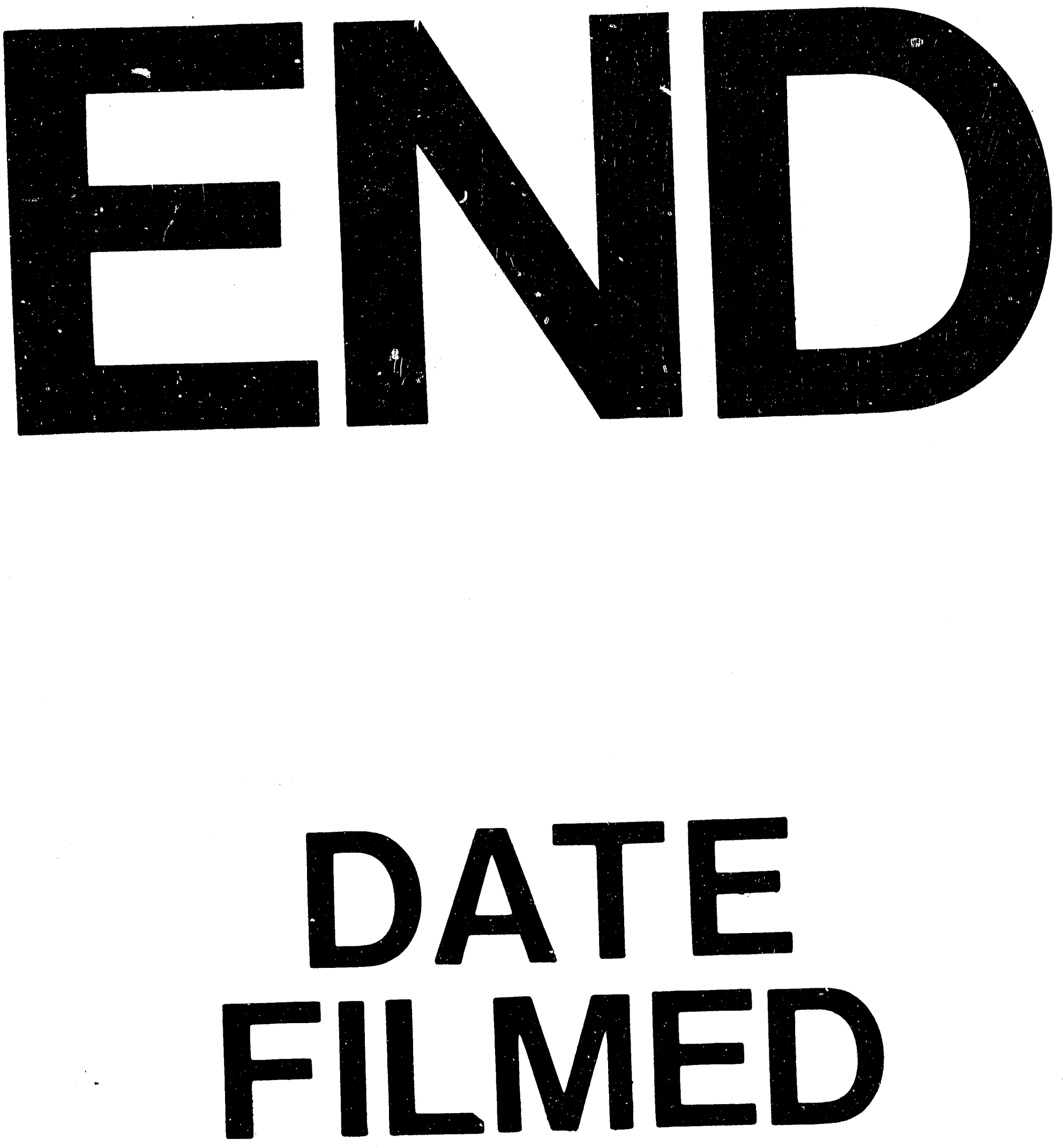

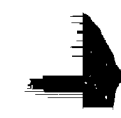

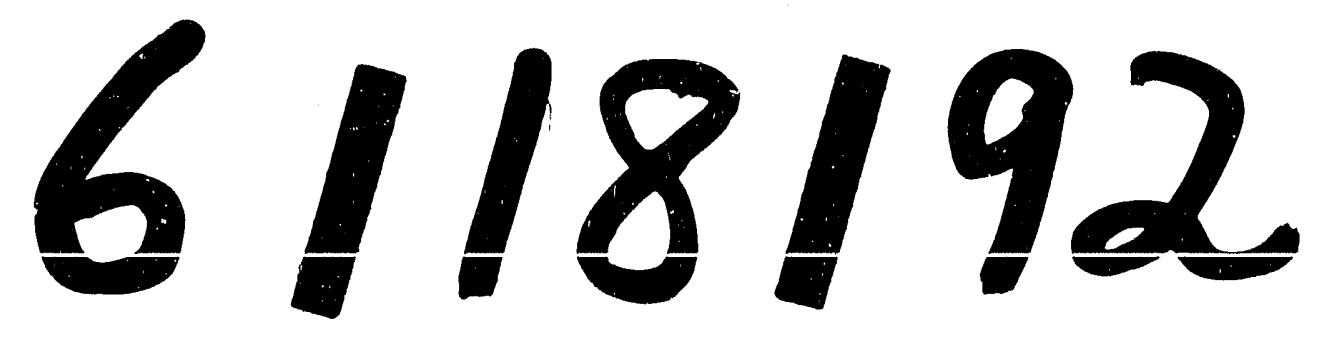


